\title{
Design of flyer-plate-driven compressible turbulent mix experiments using $Z$
}

\author{
R. Paul Drake \\ Atmospheric Oceanic and Space Sciences, University of Michigan, 2455 Hayward Street, Ann Arbor, \\ Michigan 48109
}

(Received 28 December 2001; accepted 20 May 2002)

\begin{abstract}
The use of flyer plates to drive compressible turbulent mix experiments is discussed. The experimental parameters can be optimized, in order to maximize the degree of nonlinear development of either the Rayleigh-Taylor or the Richtmyer-Meshkov instability. Analytic formulas are presented for this optimization. Results of this analysis and of simulations are shown for experiments that might be accomplished on the $\mathrm{Z}$ machine at Sandia National Laboratories [M. K. Matzen, Phys. Plasmas 4, 1519 (1997)]. One finds that unique experiments could be accomplished with this device. (C) 2002 American Institute of Physics. [DOI: 10.1063/1.1493209]
\end{abstract}

\section{INTRODUCTION}

In studies of compressible turbulent mixing, it has been an enduring challenge to follow, in a strongly shocked, highly compressible medium, the evolution of instabilities from their early nonlinear phases through the development of a fully turbulent state. Recent experimental advances offer the possibility of driving such unstable phenomena much further into the nonlinear regime has been possible previously. Here we consider the optimization of experiments that use flyer plates to study compressible turbulent mixing. The advent of high-velocity $(>20 \mathrm{~km} / \mathrm{s})$, solid state flyers ${ }^{1,2}$ on the $\mathrm{Z}$ machine ${ }^{3}$ at Sandia National Laboratories, along with the activation of $\mathrm{x}$-ray backlighting, provides a direct motivation for the discussion here. $\mathrm{Z}$ launches the flyer plates by driving a current through a thin layer of conducting material, causing the $\mathbf{J} \times \mathbf{B}$ force to accelerate the flyer toward its target. This technique makes possible very clean experiments. Here we consider the problem of designing of an optimized hydrodynamic instability experiment using flyer plates, taking specific parameters from $\mathrm{Z}$.

We will discuss the optimization of a flyer-driven experiment for either Rayleigh-Taylor ${ }^{4,5}$ (RT) or Richtmyer-Meshkov ${ }^{6,7}$ (RM) experiments. The RT instability develops when the acceleration of an interface is such that a less-dense fluid is pushing on a more dense fluid. The interface itself may accelerate or decelerate. For example, RT occurs during the acceleration of dense plasma by laserheated underdense plasma in inertial fusion targets, and also during the deceleration of denser layers by less-dense layers during the explosion of a star. RT experiments that begin with strong shocks operate by decelerating the interface between the material that is initially shocked and a subsequent, less-dense material. In this RT case, one wants to decelerate the interface immediately after it is shocked (with minimum coasting time), and to move the interface as far as possible. ${ }^{8}$ The RM instability develops when a structured interface is shocked. The structure introduces nonplanar velocity components at the interface, with the consequence that the interface becomes more structured with time. In RM experiments, one wants to cause the interface to coast steadily after it is shocked, for the longest possible time (so that RT growth does not confuse the interpretation). In both cases, what matters in an experiment (to a first approximation ${ }^{8}$ ) is the ratio of the distance the interface moves to the wavelength of the perturbation from which the instability develops. In practical terms, the degree to which a given experiment can follow the nonlinear evolution of the unstable interface is measured by the ratio of this distance the interface moves to the spatial resolution of the diagnostics. We present here an analysis and analytic relations that can guide the achievement of these goals, and hydrodynamic simulations showing what one can do using flyer plates on $\mathrm{Z}$.

Both RM and RT instabilities have been studied extensively in gasses and in liquids. Our focus here, however, is the study of these mechanisms in plasmas, at interfaces that are strongly shocked. Prior work of this type has always involved the use of lasers to produce the strong shocks. There has been extensive work on the RT instability, mostly at ablatively accelerated surfaces (including early work reviewed by Kilkenny ${ }^{9}$ and more recent work ${ }^{10-15}$ ), but also at embedded interfaces. ${ }^{16,17}$ In such work, it has proven feasible to shock an interface to high velocity (typically above 10 $\mathrm{km} / \mathrm{s}$ ), and then to decelerate it over a distance sometimes approaching $1 \mathrm{~mm}$. This distance is limited in two ways in the laser experiments. First, edge effects typically prevent one from pushing an interface much farther than the diameter of the experimental package. By that point the effects of the edges of the package typically propagate inward so that the behavior becomes more complicated and no longer represents the pure evolution of the RT instability. Laser experiments that use a few $\mathrm{kJ}$ of laser energy to drive strong shocks through solid materials are limited to spot diameters below 1 $\mathrm{mm}$. Second, the total available energy limits the maximum distance an interface will travel. In any system in which an unstable interface is shocked by a blast wave that proceeds beyond it into low-density matter, the interface eventually decelerates to rest (after which it very slowly begins to move backwards with the material flowing away from the shock). 
Here again, interfaces driven by lasers that can apply a few $\mathrm{kJ}$ of energy to a sub-mm spot, in an otherwise viable experiment, typically travel a mm or so.

Prior work on the RM instability has including pioneering experiments by Dimonte et al. ${ }^{18,19}$ Further experiments on RM, not yet published, are underway at the Omega laser. ${ }^{20}$ In some of these latter experiments, ${ }^{21}$ the interface is made to coast at constant velocity for about $1 / 3 \mathrm{~mm}$. This distance is limited by one-dimensional shock dynamics, established by the duration over which one can drive a steady shock. The times in the experiment, including those required for rarefactions to overtake shocks so they are no longer steady, are proportional to this duration. These times are typically limited to $<10 \mathrm{~ns}$ in a laser experiment, which also requires a shock velocity of tens of $\mathrm{km} / \mathrm{s}$ (=tens of $\mu \mathrm{m} / \mathrm{ns}$ ) to produce a strong shock in useful materials. The resulting, initial shocked region is a few hundred microns long, leading to a maximum steady interface motion of a few hundred microns.

We will see below that $\mathrm{Z}$ has the potential to greatly exceed the distances over which the interface was moved in these laser experiments. In both the experiments with lasers and those with $\mathrm{Z}$, the principal diagnostic is side-on $\mathrm{x}$-ray backlighting. Here by "side-on" one means in a direction transverse to the direction of shock propagation. Face-on backlighting, extremely useful during past experiments to explore the linear phases of instability evolution, becomes much less useful once the spike tips broaden significantly. The diagnostic resolution on $\mathrm{Z}$ should approach that of the laser experiments, since $\mathrm{Z}$ will also use laser-driven $\mathrm{x}$-ray backlighting to diagnose the experiments. This should enable experiments using $\mathrm{Z}$ to follow the instability evolution much further into the nonlinear regime.

For completeness, one should note that any large laser can shock and accelerate a slab of material, producing a "plasma flyer" that can deliver energy and momentum to a desired target. If the laser is large enough $(\sim 1 \mathrm{~kJ})$, then the plasma flyer can have sufficient lateral size to permit studies of mixing. Actual laser experiments, however, have typically chosen to take the simpler approach of using the laser directly to drive a shock wave into a chosen medium.

In the following, we first develop a common framework for the description of strong shocks driven by flyer plates, and discuss some of the properties of such systems. We next consider the optimization of RT experiments. After that, we discuss RM experiments. The examples use flyer plate properties that have been achieved on Z. ${ }^{1,2}$ Specifically, we assume Al flyer plates, $350 \mu \mathrm{m}$ thick, launched at $21 \mathrm{~km} / \mathrm{s}$ using $\mathbf{J} \times \mathbf{B}$ forces. We note that these flyers are believed to be in the solid state and that their transverse dimensions are $>10 \mathrm{~mm}$. This implies that $\mathrm{cm}$-scale experiments can be undertaken without fear that they will be greatly compromised by multidimensional effects. (Indeed, the limitations on $\mathrm{Z}$ are likely to be diagnostic ones, as discussed below.) Throughout the following, the simulations shown are from the Lagrangian radiation-hydrodynamics code, HYADES, ${ }^{22}$ run with SESAME equation of state tables. In the experiments discussed, however, neither radiation nor heat conduction is important.

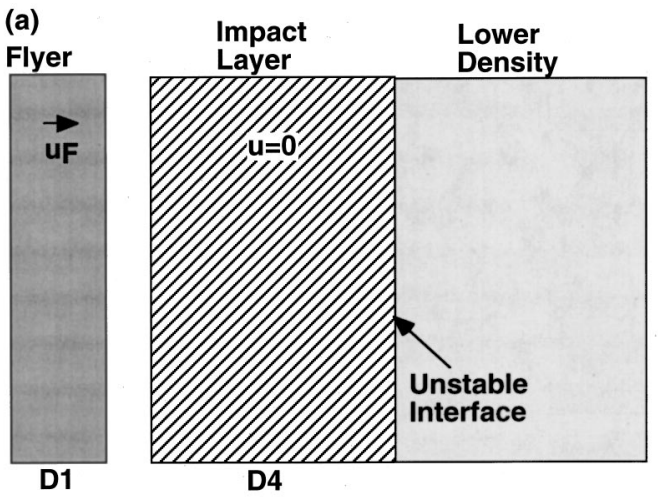

(b)

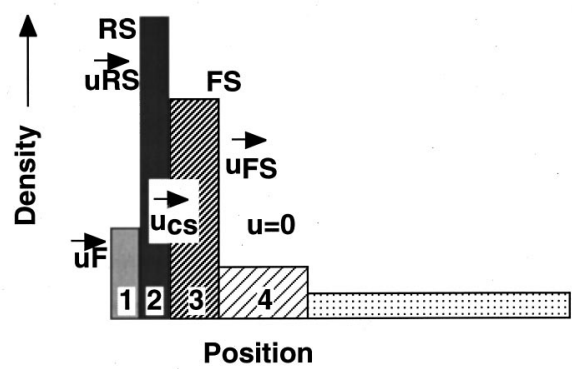

FIG. 1. Schematic and structure of the flyer plate experiment. (a) Schematic showing the flyer plate about to impact an impact layer, adjacent to a lowerdensity layer. (b) Density profile showing the definitions used in the analysis.

\section{FLYER PLATE DRIVEN SHOCKS}

Because both the RT and the RM problem involve the use of a flyer that strikes an impact layer, it is worthwhile to adopt some common terminology and to identify some standard relationships for such a system. We will describe the evolution of flyer-plate driven systems using the geometry shown in Fig. 1. Figure 1(a) shows a schematic of the experiment. The aluminum flyer is about to strike the impact layer. The impact layer typically might be made of plastic to allow the use of a doped layer for diagnostic purposes. The unstable interface is at the boundary of the impact layer and a lower-density material, such as a $\mathrm{CH}$ foam. Figure 1(b) shows the density profile during the initial phase of the experiment, and serves to define several quantities of interest for our analysis. Let $D, \rho, c$, and $u$ represent thickness, density, sound speed, and velocity, respectively. As in Fig. 1(b), we will refer to the unshocked flyer as region 1 and the unshocked impact layer as region 4, with the corresponding shocked regions being 2 and 3, using subscripts to designate the regions. In the lab frame, designate velocities by $u$ with subscripts RS, FS, CS, and F to identify the reflected shock, forward shock, contact surface, and initial undisturbed flyer plate, respectively. In addition, assume that the material in unshocked region $j$ has a polytropic index, $\gamma_{j}$. (For very strongly shocked materials, it is frequently a reasonable assumption to take $\gamma=5 / 3$.) Assume that the flyer and the impact layer are both initially cold, and that all shocks are strong. One then finds, by applying standard formulas, that 


$$
\begin{aligned}
u_{\mathrm{CS}}= & \left(1+\sqrt{\frac{\left(\gamma_{4}+1\right) \rho_{4}}{\left(\gamma_{1}+1\right) \rho_{1}}}\right)^{-1} u_{F}, \\
u_{\mathrm{RS}}= & \left(1-\frac{\gamma_{1}-1}{2} \sqrt{\frac{\left(\gamma_{4}+1\right) \rho_{4}}{\left(\gamma_{1}+1\right) \rho_{1}}}\right) \\
& \times\left(1+\sqrt{\frac{\left(\gamma_{4}+1\right) \rho_{4}}{\left(\gamma_{1}+1\right) \rho_{1}}}\right)^{-1} u_{F}
\end{aligned}
$$

and

$$
u_{\mathrm{FS}}=\frac{\gamma_{4}+1}{2}\left(1+\sqrt{\frac{\left(\gamma_{4}+1\right) \rho_{4}}{\left(\gamma_{1}+1\right) \rho_{1}}}\right)^{-1} u_{F} .
$$

Note that the contact surface velocity is half the flyer velocity when the impact layer is of the same material as the flyer, and increases to the flyer velocity as the impact layer density approaches zero. It is also helpful to know the velocity of the reverse shock in the frame where the flyer plate velocity is zero, $u_{\mathrm{RS}}^{\prime \prime}$, which is

$$
\begin{aligned}
u_{\mathrm{RS}}^{\prime \prime}= & -\sqrt{\frac{\left(\gamma_{4}+1\right) \rho_{4}}{\left(\gamma_{1}+1\right) \rho_{1}}}\left(1+\sqrt{\frac{\left(\gamma_{4}+1\right) \rho_{4}}{\left(\gamma_{1}+1\right) \rho_{1}}}\right)^{-1} \\
& \times \frac{\gamma_{1}+1}{2} u_{F} .
\end{aligned}
$$

The sound speeds in the shocked material are

$$
\begin{aligned}
c_{3} & =\sqrt{\frac{\gamma_{4}\left(\gamma_{4}-1\right)}{2}} u_{\mathrm{CS}} \\
& =\sqrt{\frac{\gamma_{4}\left(\gamma_{4}-1\right)}{2}}\left(1+\sqrt{\frac{\left(\gamma_{4}+1\right) \rho_{4}}{\left(\gamma_{1}+1\right) \rho_{1}}}\right)^{-1} u_{F},
\end{aligned}
$$

and

$$
\begin{aligned}
c_{2}= & \sqrt{\frac{\gamma_{1}\left(\gamma_{1}-1\right)}{2}}\left(u_{F}-u_{\mathrm{CS}}\right) \\
= & \sqrt{\frac{\gamma_{1}\left(\gamma_{1}-1\right)}{2}} \sqrt{\frac{\left(\gamma_{4}+1\right) \rho_{4}}{\left(\gamma_{1}+1\right) \rho_{1}}} \\
& \times\left(1+\sqrt{\left.\frac{\left(\gamma_{4}+1\right) \rho_{4}}{\left(\gamma_{1}+1\right) \rho_{1}}\right)^{-1} u_{F} .}\right.
\end{aligned}
$$

One can calculate the pressure on the shocked layer (regions 2 and 3) from the above equations. Assuming that $\gamma=5 / 3$, this approaches 8 Mbars when $\mathrm{Al}$ impacts $\mathrm{Al}$, and decreases as the impact layer density decreases, being about 1 Mbar when $\rho_{1} / \rho_{4}$ is 10 and 200 kbars when $\rho_{1} / \rho_{4}$ is 100 .

\section{RAYLEIGH-TAYLOR EXPERIMENTS}

In this section we consider how to optimize flyer plate experiments to study the RT instability. One desires, for this purpose, to shock the interface to as large a velocity as possible, but then to immediately begin to decelerate it. This will minimize the coasting period during which RM will grow. One then desires to push the decelerating interface through as large a distance as one can. ${ }^{8}$ This maximizes the nonlinear evolution of the instability. We now address the problem of how to accomplish these ends.

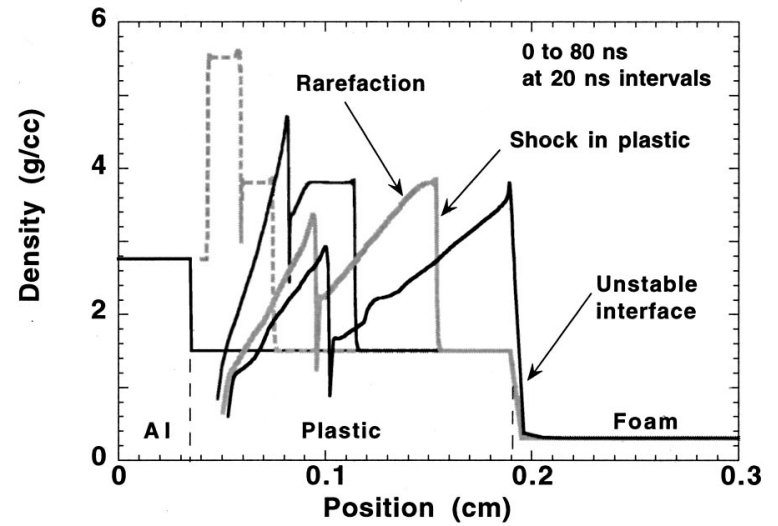

FIG. 2. The density profile during the initial phases of an experiment in a system optimized for Rayleigh-Taylor experiments. An Al flyer plate, 0.35 $\mathrm{mm}$ thick, of density $2.76 \mathrm{~g} / \mathrm{cm}^{3}$, and moving at $20 \mathrm{~km} / \mathrm{s}$, impacts a 1.55 $\mathrm{g} / \mathrm{cm}^{3}$ plastic layer that is $1.55 \mathrm{~mm}$ thick. To the right of the plastic is a $\mathrm{CH}$ foam layer of density $300 \mathrm{mg} / \mathrm{cm}^{3}$. Profiles are shown at $20 \mathrm{~ns}$ intervals from 0 to $80 \mathrm{~ns}$.

The key here is to accelerate the interface by a blast wave as opposed to an extended shock. Here, by a blast wave we mean a structure in which a shock front is followed immediately by a zone of decreasing velocity, pressure, and density. In contrast, an extended shock is a structure in which the shock front is followed by a region of uniform fluid flow. Figure 2 shows how one can achieve such a blast wave in a flyer plate experiment. (The calculations that produced Fig. 2 are discussed further below.) The figure shows density profiles at five successive times. The leftmost profile shows the undisturbed densities, at the moment when the dense Al flyer plate is just striking the less-dense plastic layer. The unstable interface of interest is at the rightmost edge of the plastic, beyond which is lower-density foam. The impact of the flyer plate produces shock waves that travel into the flyer plate and into the impacted material, seen in the next (dashed, gray) profile. For this RT case, one makes the impacted material quite thick. The reflected rarefaction wave in the flyer plate then reaches the shocked plastic long before the shock wave in the plastic reaches the end of the plastic, as the third profile in Fig. 1 shows. The rarefaction in the plastic will eventually approach and overtake the shock in the plastic, as the fourth and fifth profiles show. To optimize the experiment, one wants this to occur just as they both reach the rear surface. If the rarefaction arrives sooner, then the blast wave will be weaker than it could be when it reaches the unstable interface. If it arrives later, then the interface will coast after it is shocked and before it begins to decelerate.

It also makes sense, in such an experiment, to locate the unstable interface at the rear surface of the impact material. The flyer plate is typically a conductive material such as Al, at least in Z-pinch systems, through which X-ray backlighting is quite difficult. For this reason, as is discussed further below, one will typically want to use an impact-layer material that differs from the flyer-plate material.

We can specify the required system parameters mathematically, as follows. The time after impact it takes for the forward shock to reach the rear of the impact layer, $t_{4}$, is 


$$
t_{4}=\frac{D_{4}}{u_{\mathrm{FS}}}=\frac{D_{4}}{u_{F}} \frac{2}{\gamma_{4}+1}\left(1+\sqrt{\frac{\left(\gamma_{4}+1\right) \rho_{4}}{\left(\gamma_{1}+1\right) \rho_{1}}}\right)
$$

and the time after impact that it takes for the rarefaction to reach the same surface, $t_{1}$, is

$$
t_{1}=\frac{D_{1}}{\left|u_{\mathrm{RS}}^{\prime \prime}\right|}+\frac{\gamma_{1}-1}{\gamma_{1}+1} \frac{D_{1}}{c_{2}}+\frac{\gamma_{4}-1}{\gamma_{4}+1} \frac{D_{4}}{c_{3}}
$$

where the three terms identify the time it takes the shock to reach the front surface of the flyer, the time it takes the rarefaction wave to cross the shocked flyer material, and the time it takes the rarefaction wave to cross the shocked impact layer, respectively. Substituting for the speeds, one finds

$$
\begin{aligned}
t_{1}= & \frac{D_{1}}{u_{F}} \frac{2}{\gamma_{1}+1}\left(1+\sqrt{\frac{\left(\gamma_{4}+1\right) \rho_{4}}{\left(\gamma_{1}+1\right) \rho_{1}}}\right) \\
& \times\left\{\sqrt{\frac{\left(\gamma_{1}+1\right) \rho_{1}}{\left(\gamma_{4}+1\right) \rho_{4}}}\left[1+\sqrt{\frac{\left(\gamma_{1}-1\right)}{2 \gamma_{1}}}\right]\right. \\
& \left.+\frac{\left(\gamma_{1}+1\right)}{\left(\gamma_{4}+1\right)} \sqrt{\frac{\left(\gamma_{4}-1\right)}{2 \gamma_{4} D_{4}}}\right\} .
\end{aligned}
$$

Setting these two times equal, one finds the optimum ratio of the impact layer thickness to the flyer thickness,

$$
\frac{D_{4}}{D_{1}}=\sqrt{\frac{\rho_{1}}{\rho_{4}}} \sqrt{\frac{\left(\gamma_{1}+1\right)}{\left(\gamma_{4}+1\right)}} \frac{\left(1+\sqrt{\left(\gamma_{1}-1\right) / 2 \gamma_{1}}\right)}{\left(1-\sqrt{\left.\left(\gamma_{4}-1\right) / 2 \gamma_{4}\right)}\right.} .
$$

For $\gamma_{1}=\gamma_{4}=5 / 3$, this ratio is $2.62 \sqrt{\rho_{1} / \rho_{4}}$. If, for example, we use a $\left(1.55 \mathrm{~g} / \mathrm{cm}^{3}\right)$ plastic material for the impact layer that is just over half the density of an aluminum flyer plate, then an estimate of the ideal impact layer thickness is 3.5 times the thickness of the flyer plate.

One can see the formation of the blast wave and the behavior of the interface in the results of one-dimensional simulations shown in Figs. 2 and 3. The corresponding first estimate of the impact layer thickness, from Eq. (10), would be $1.225 \mathrm{~mm}$, although seeking the optimum timing in simulations leads one to increase this to $1.55 \mathrm{~mm}$. To optimize the actual thickness in the experiment, one would have to vary this thickness while examining the behavior of a planar interface.

The density of the low-density material determines how far the interface moves. As material accumulates, the interface decelerates until it eventually stops and reverses direction. In the asymptotic limit, the blast wave decelerates as a power law of time, but the accumulation of material causes the interface to decelerate more quickly. Figure 3 shows the deceleration of the interface, for the system of Fig. 2, if the low-density material is at $300 \mathrm{mg} / \mathrm{cm}^{3}$. One can see that the interface slows down as the blast wave moves to the right, and that the distance between them increases with time.

Figure 4 shows how the position and velocity of the interface evolves in time. One can see that, during $1.5 \mu \mathrm{s}$, the interface moves about $5 \mathrm{~mm}$ as it decelerates from nearly 15 $\mathrm{km} / \mathrm{s}$ to about $2 \mathrm{~km} / \mathrm{s}$. This motion is much larger than that which can be obtained in existing laser experiments, and one would correspondingly expect the instability to progress much further into the nonlinear regime. The instantaneous
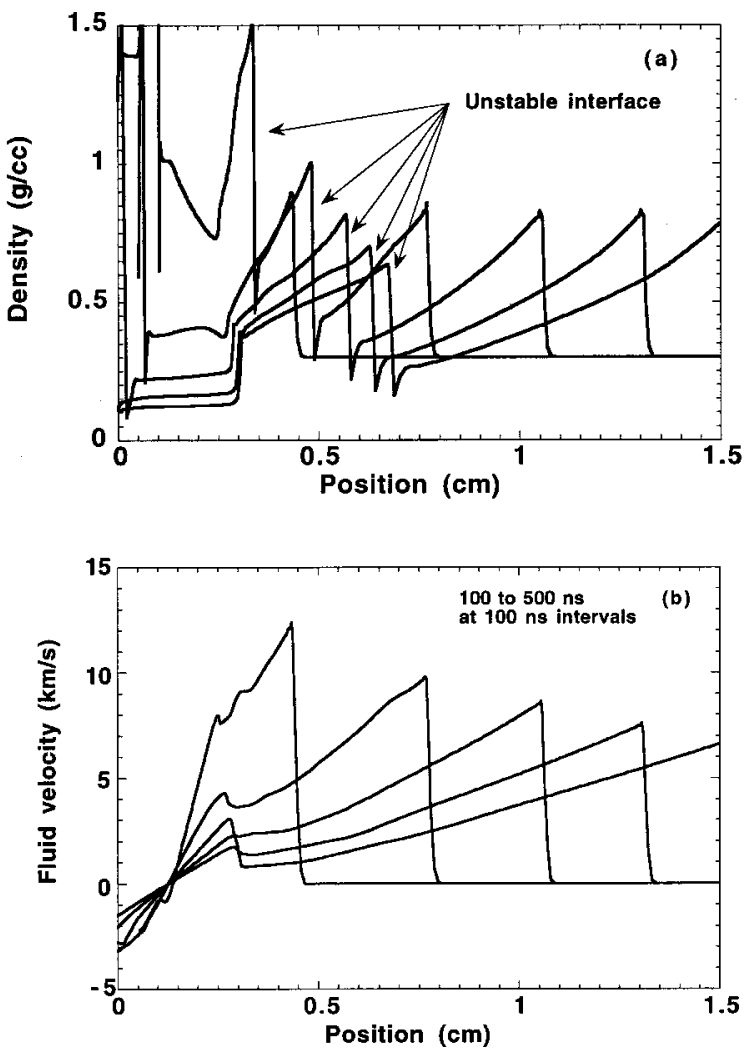

FIG. 3. The density and velocity profiles during the later phases of the experiment of Fig. 1. Profiles are shown at $100 \mathrm{~ns}$ intervals from 100 to 500 ns.

RT growth rate is $\gamma_{\mathrm{RT}}=\sqrt{A k g}$, in which the Atwood number $A=\left(\rho_{1}-\rho_{4}\right) /\left(\rho_{1}+\rho_{4}\right), g$ is the deceleration, and $k$ is the wave number. For $A=0.53$, corresponding to Fig. 3, one can numerically find the growth exponent $G=\int \gamma_{\mathrm{RT}} d t$ for the case shown. For a perturbation with a $100 \mu \mathrm{m}$ wavelength, one finds $G=21.6$. If the growth were to be linear (which it will not), this would correspond to an overall growth factor of $\mathrm{e}^{G}=2 \times 10^{9}$.

We now consider the limitations on such an experiment. Given that the lateral scale of the flyer can approach $1 \mathrm{~cm}$, but that we can only move the interface several $\mathrm{mm}$, edge effects are not the primary limitation. Instead, diagnostic is-

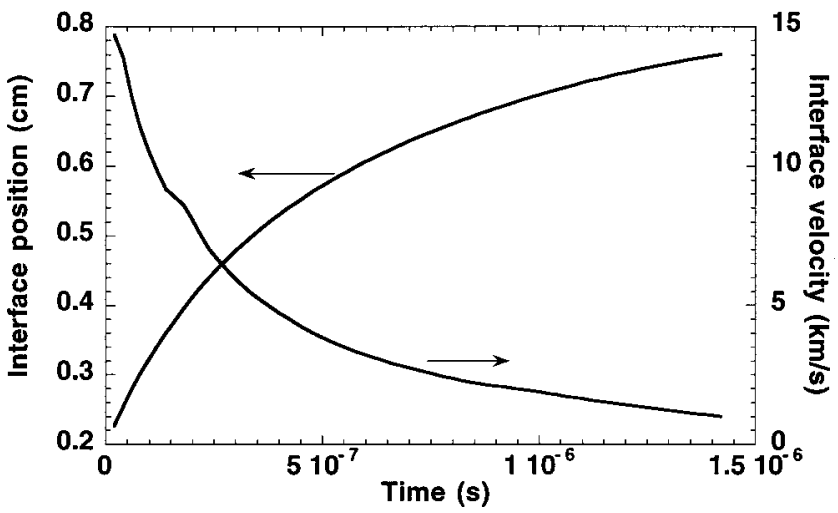

FIG. 4. The position and velocity of the unstable interface are shown vs time, for the experiment of Fig. 2. 
sues predominate. One specific viable experiment would use plastic as the impact layer material, followed by a lowdensity foam of density $50-100 \mathrm{mg} / \mathrm{cm}^{3}$, in a package having a lateral dimension of 5-10 $\mathrm{mm}$. In the resulting density profile, the plastic would be quite opaque to $\mathrm{Fe} K_{\alpha}$ x rays at $6.7 \mathrm{keV}$, while the foam would be adequately transparent. A better experiment would use an impact layer material through which one can use $\mathrm{x}$-ray backlighting and within which one can locate an absorptive diagnostic layer, to obtain a more local sample of the evolving structures. Several plastics can be used in this way. However, it would be difficult to obtain enough signal with present-day backlighting methods because of the large $\mathrm{x}$-ray absorption by the plastic, which will have a density near $1 \mathrm{~g} / \mathrm{cm}^{3}$ after the interface has moved several $\mathrm{mm}$. For example, the transmission of an Fe $K_{\alpha}$ backlighter through several $\mathrm{mm}$ of plastic at this density is a few percent. There is some chance that the increased signal enabled by spherical crystal imaging ${ }^{23,24}$ would make such an approach feasible. Alternatively, such experiments will require the advent of brighter or higher-energy backlighters, perhaps achievable by the conversion of the $\mathrm{Z}$ backlighter to higher-intensity, short-pulse operation. Another approach, which would work with present-day backlighting methods, would be to use a foam impact layer followed by a layer of even-lower-density foam. This would require that one use sandwiches of different foam materials (or develop the ability to locally dope foam), in order to obtain the local absorptive layer.

\section{RICHTMYER-MESHKOV EXPERIMENTS}

In producing the RM instability, one would like to abruptly accelerate an interface to a constant velocity and then to sustain the steady drift of this interface for as long as possible. For the (easier) case of an interface at which the density decreases, the motion of the interface occurs while a rarefaction wave propagates backward into the denser material. Thus, the problem becomes one of maximizing the duration of the steady rarefaction. For the flyer/impact-layer problem, the rarefaction of the impact layer will remain steady until it is disturbed, either by meeting the rarefaction from the flyer or by reaching the contact surface between the flyer and the impact layer. After that, a pressure and velocity decrease will propagate forward to the unstable interface, disturbing it when the interface is reached. Thus, the design goal is to achieve maximum motion of the interface before this occurs. One can show that the maximum undisturbed motion of the interface is achieved by choosing the thickness of the impact layer so that the rarefactions in the flyer plate and in the impact layer arrive simultaneously at the contact surface. If the impact layer is thinner than necessary to achieve this, then the rarefaction from the unstable interface reaches the contact surface sooner than need be. If the impact layer is thicker, then the rarefaction from the flyer moves through the shocked impact layer material faster than the shock wave did initially. This turns out to reduce the time available for undisturbed motion of the unstable interface.

We can express these relationships mathematically to estimate the optimum ratio of impact layer thickness to flyer
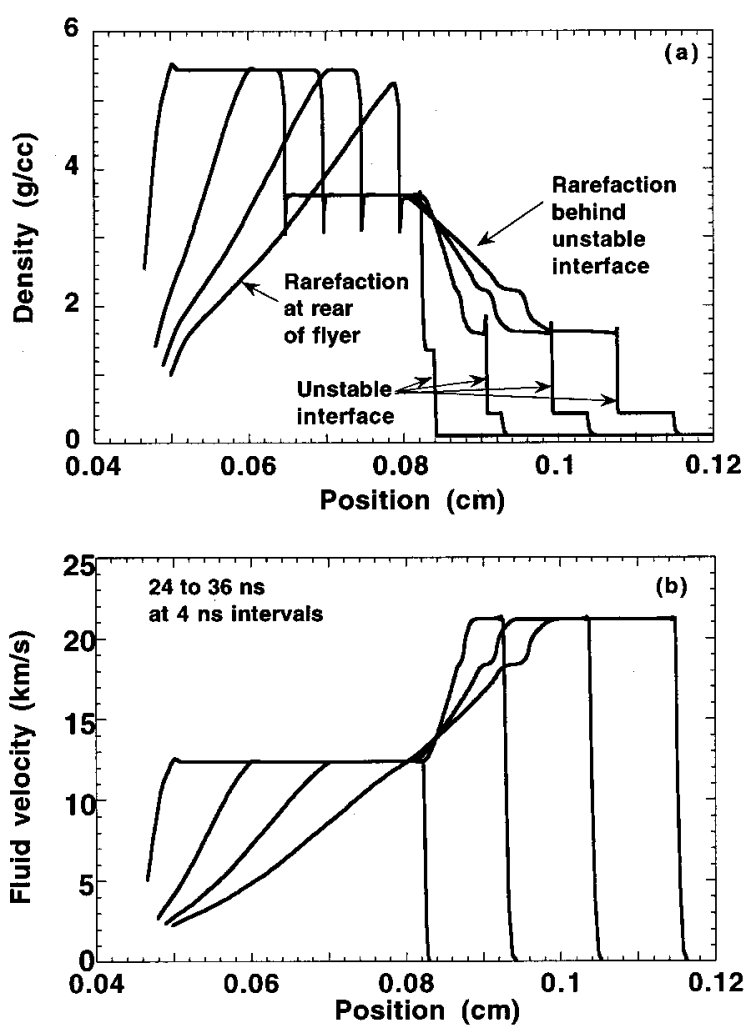

FIG. 5. The density and velocity profiles during the development of the rarefactions in a RM experiment.

plate thickness. The arrival time of the rarefaction in the flyer is

$$
\begin{aligned}
t_{1}= & \frac{D_{1}}{\left|u_{\mathrm{RS}}^{\prime \prime}\right|}+\frac{\gamma_{1}-1}{\gamma_{1}+1} \frac{D_{1}}{c_{2}}=\frac{D_{1}}{u_{F}}\left(1+\sqrt{\frac{\left(\gamma_{1}+1\right) \rho_{1}}{\left(\gamma_{4}+1\right) \rho_{4}}}\right) \\
& \times\left(\frac{2}{\gamma_{1}+1}+\sqrt{\frac{2}{\gamma_{1}\left(\gamma_{1}-1\right)}}\right),
\end{aligned}
$$

in which there are terms expressing the traversal of the flyer by the reflected shock and then by the forward rarefaction. The arrival time of the rarefaction at the contact surface in the impact layer is

$$
\begin{aligned}
t_{4}= & \frac{D_{4}}{u_{\mathrm{FS}}}+\frac{\gamma_{4}-1}{\gamma_{4}+1} \frac{D_{4}}{c_{3}}=\frac{D_{4}}{u_{F}}\left(1+\sqrt{\frac{\left(\gamma_{4}+1\right) \rho_{4}}{\left(\gamma_{1}+1\right) \rho_{1}}}\right) \\
& \times\left\{\frac{2}{\gamma_{4}+1}+\sqrt{\frac{2}{\gamma_{4}\left(\gamma_{4}-1\right)}}\right\},
\end{aligned}
$$

in which there are terms expressing the traversal of the impact layer by the forward shock and then by the rearward rarefaction. Setting these equal, one finds

$$
\begin{aligned}
\frac{D_{4}}{D_{1}}= & \sqrt{\frac{\rho_{1}}{\rho_{4}}} \sqrt{\frac{\left(\gamma_{1}+1\right)}{\left(\gamma_{4}+1\right)}} \\
& \times \frac{\left[\left(2 /\left(\gamma_{1}+1\right)\right)+\sqrt{2 /\left(\gamma_{1}\left(\gamma_{1}-1\right)\right)}\right]}{\left[\left(2 /\left(\gamma_{4}+1\right)\right)+\sqrt{2 /\left(\gamma_{4}\left(\gamma_{4}-1\right)\right)}\right.} .
\end{aligned}
$$



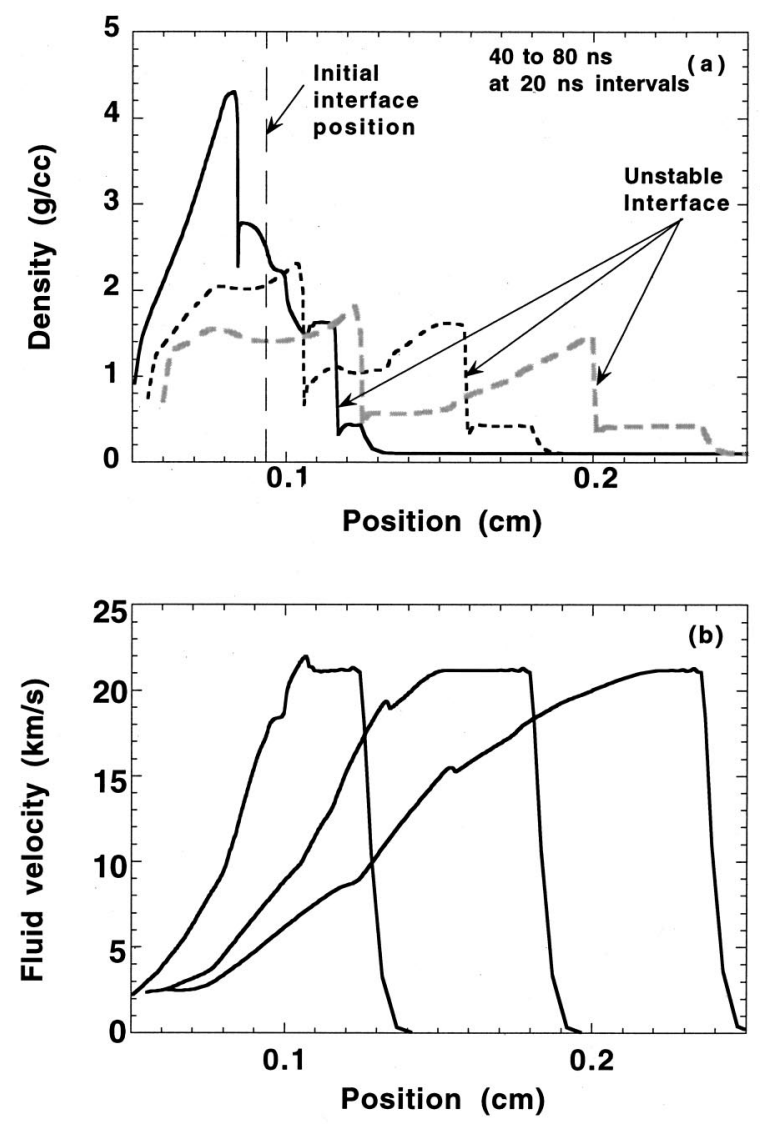

FIG. 6. The density and velocity profiles during the steady motion of the unstable interface in the RM experiment.

For an $\mathrm{Al}$ flyer and a plastic impact layer of half its density, and for $\gamma_{1}=\gamma_{4}=5 / 3$, one obtains $D_{4} / D_{1}=1.4$. Here again we can simulate this to observe the behavior of the unstable interface.

Figures 5 and 6 show the results of simulations for this case. Figure 5 shows the development of the rarefactions. Here an Al flyer plate impacts a plastic layer of half its density $\left(1.35 \mathrm{~g} / \mathrm{cm}^{3}\right)$, for which Eq. (13) would suggest that the plastic thickness should be 1.4 times the Al thickness. This is the case in Fig. 5. The plastic thickness is $490 \mu \mathrm{m}$, which works quite well. The forward and rearward rarefactions meet at the contact surface at $36 \mathrm{~ns}$, by which time the unstable interface has been moving for about $10 \mathrm{~ns}$ and has moved about $250 \mu \mathrm{m}$. Figure 6 shows that the unstable interface continues to move for an additional $40 \mathrm{~ns}$ before it is overtaken by the developing deceleration. By this time the interface has moved about $1 \mathrm{~mm}$, which is three times the distance that can be achieved by similar methods in presentday laser experiments.

The RM experiments on $\mathrm{Z}$ are limited by the onedimensional shock dynamics just described. In contrast to the RT case, the diagnostic limitations are not severe. One would employ a plastic impact layer that included a strip of absorptive material for diagnostic purposes. An $\mathrm{Fe} K_{\alpha}$ backlighter would work well, so long as the lateral extent of the package did not exceed 1.5-2 $\mathrm{mm}$. As the unstable interface does not move even this far, such a design would be viable.

\section{CONCLUSION}

In this paper, we have discussed the problem of designing hydrodynamic instability experiments driven by flyer plates. We have presented analytical formulas that can be used to obtain an estimate of the optimum parameters for either RT or RM experiments. In each case, we have shown optimized simulations of experiments that would be possible today on the $\mathrm{Z}$ machine. In both cases, $\mathrm{Z}$ has the potential to access unique regimes of hydrodynamic instability, by pushing the RT or RM instability much further into the nonlinear regime than can be accomplished in any other existing research facilities. Before concluding, however, it is worth emphasizing that the various research facilities have important complementary capabilities. The laser facilities offer the advantages of a much higher experiment rate and of more flexible diagnostics than the $\mathrm{Z}$ pinch can offer, enabling much more detailed scaling studies. The $\mathrm{Z}$ pinch has the advantage that it can push the hydrodynamic instabilities, in a strongly shocked plasma medium, much farther into their nonlinear state.

\section{ACKNOWLEDGMENTS}

The author acknowledges useful discussions of this topic with Marcus Knudsen, Jim Asay, Chris Deeney, and Tom Melhorn of the Sandia National Laboratories and with Gail Glendinning and Jave Kane of the Lawrence Livermore National Laboratories.

This work was supported by the Sandia National Laboratories and by the High Energy Density grants program of the U.S. Department of Energy.

${ }^{1}$ C. A. Hall, J. R. Asay, M. D. Knudson, W. A. Stygar, R. B. Spielman, T. D. Pointon, D. B. Reisman, A. Toor, and R. C. Cauble, Rev. Sci. Instrum. 72, 3587 (2001).

${ }^{2}$ M. D. Knudson, D. L. Hanson, J. E. Bailey, C. A. Hall, J. R. Asay, and W. W. Anderson, Phys. Rev. Lett. 87, 225501 (2001).

${ }^{3}$ M. K. Matzen, Phys. Plasmas 4, 1519 (1997).

${ }^{4}$ Lord Rayleigh, Scientific Papers II (Cambridge, England, 1900).

${ }^{5}$ S. G. Taylor, Proc. R. Soc. London, Ser. A 201, 192 (1950).

${ }^{6}$ D. H. Richtmyer, Commun. Pure Appl. Math. 13, 297 (1960).

${ }^{7}$ E. E. Meshkov, Fluid Dyn. 4, 101 (1969).

${ }^{8}$ R. P. Drake and P. A. Keiter, Phys. Plasmas 9, 382 (2002).

${ }^{9}$ J. D. Kilkenny, S. G. Glendinning, S. W. Haan, B. A. Hammel, J. D. Lindl, J. P. Knauer, and C. P. Verdon, Phys. Plasmas 1, 1379 (1994).

${ }^{10}$ B. A. Remington, S. V. Weber, M. M. Marinak, S. W. Haan, J. D. Kilkenny, R. J. Wallace, and G. Dimonte, Phys. Plasmas 2, 241 (1995).

${ }^{11}$ K. Shigemori, H. Azechi, M. Nakai, M. Honda, K. Meguro, N. Miyanaga, H. Takabe, and K. Mima, Phys. Rev. Lett. 78, 250 (1997).

${ }^{12}$ S. G. Glendinning, S. N. Dixit, B. A. Hammel, D. H. Kalantar, M. H. Key, J. D. Kilkenny, J. P. Knauer, D. M. Pennington, B. A. Remington, R. J. Wallace, and S. V. Weber, Phys. Rev. Lett. 78, 3318 (1997).

${ }^{13}$ W. W. Hsing, C. W. Barnes, J. B. Beck, N. M. Hoffman, D. Galmiche, A. Richard, J. Edwards, P. Graham, S. Rothman, and B. Thomas, Phys. Plasmas 4, 1832 (1997).

${ }^{14}$ J. P. Knauer, R. Betti, D. K. Bradley, T. R. Boehly, T. J. B. Collins, V. N. Goncharov, P. W. McKenty, D. D. Meyerhofer, V. A. Smalyuk, C. P. Verdon, S. G. Glendinning, D. H. Kalantar, and R. G. Watt, Phys. Plasmas 7, 338 (2000).

${ }^{15}$ K. S. Budil, B. Lasinski, M. J. Edwards, A. S. Wan, B. A. Remington, S. V. Weber, S. G. Glendinning, L. Suter, and P. E. Stry, Phys. Plasmas 8, 2344 (2001).

${ }^{16}$ K. S. Budil, B. A. Remington, T. A. Peyser, K. O. Mikaelian, P. L. Miller, N. C. Woolsey, W. M. Wood-Vasey, and A. M. Rubenchik, Phys. Rev. Lett. 76, 4536 (1996).

${ }^{17}$ J. O. Kane, H. F. Robey, B. A. Remington, R. P. Drake, J. Knauer, D. D. 
Ryutov, H. Louis, R. Teyssier, O. Hurricane, D. Arnett, R. Rosner, and A. Calder, Phys. Rev. E 63, 055401 (2001).

${ }^{18}$ G. Dimonte and M. Schneider, Phys. Plasmas 4, 4347 (1997).

${ }^{19}$ G. Dimonte, C. E. Frerking, M. Schneider, and B. A. Remington, Phys. Plasmas 3, 614 (1996).

${ }^{20}$ T. R. Boehly, R. S. Craxton, T. H. Hinterman, J. H. Kelly, T. J. Kessler, S. A. Kumpman, S. A. Letzring, R. L. McCrory, S. F. B. Morse, W. Seka, S. Skupsky, J. M. Soures, and C. P. Verdon, Rev. Sci. Instrum. 66, 508 (1995).
${ }^{21}$ S. G. Glendinning, Lawrence Livermore National Laboratory (private communication, 2001)

${ }^{22}$ J. T. Larsen and S. M. Lane, J. Quant. Spectrosc. Radiat. Transf. 51, 179 (1994).

${ }^{23}$ Y. Aglitskiy, T. Lehecka, S. Obenschain, S. Bodner, C. Pawley, K. Gerber, J. Sethian, C. M. Brown, J. Seely, U. Feldman, and G. Holland, Appl. Opt. 37, 5253 (1998)

${ }^{24}$ Y. Aglitskiy, T. Lehecka, S. Obenschain, C. Pawley, C. M. Brown, and J. Seely, Rev. Sci. Instrum. 70, 530 (1999). 